\title{
MINI-REVIEW
}

\section{Retinoic acid inducible gene-I, more than a virus sensor}

\author{
Feng Liu, Jun Gu ${ }^{凶}$ \\ National Key Laboratory of Protein Engineering and Plant Gene Engineering, LSC, Peking University, Beijing 100871, China \\ Correspondence: gj@pku.edu.cn \\ Received March 24, 2011 Accepted April 11, 2011
}

\begin{abstract}
Retinoic acid inducible gene-I (RIG-I) is a caspase recruitment domain (CARD) containing protein that acts as an intracellular RNA receptor and senses virus infection. After binding to double stranded RNA (dsRNA) or 5'-triphosphate single stranded RNA (ssRNA), RIG-I transforms into an open conformation, translocates onto mitochondria, and interacts with the downstream adaptor mitochondrial antiviral signaling (MAVS) to induce the production of type I interferon and inflammatory factors via IRF3/7 and NF-KB pathways, respectively. Recently, accumulating evidence suggests that RIG-I could function in non-viral systems and participate in a series of biological events, such as inflammation and inflammation related diseases, cell proliferation, apoptosis and even senescence. Here we review recent advances in antiviral study of RIG-I as well as the functions of RIG-I in other fields.
\end{abstract}

KEYWORDS retinoic acid inducible gene-I (RIG-I), antiviral signaling, inflammation, innate immunity

\section{INTRODUCTION}

RIG-I (retinoic acid inducible gene-I, also known as DDX58) was first discovered and cloned as an up-regulated gene in acute promyelocytic leukemia (APL) cell line NB4 upon alltrans-retinoic acid (ATRA) stimulation (Liu et al., 2000). It encodes a protein of 925 amino acids in human, which contains an $\mathrm{N}$-terminal caspase recruitment domain (CARD), a DExD/H box helicase domain and a C-terminal repression domain (RD) (Yoneyama et al., 2004; Saito et al., 2007). RIGI, together with MDA5 (melanoma differentiation associated gene 5) and LGP2 (laboratory of genetics and physiology 2), belong to RIG-I-like receptors (RLRs). They constitute an intracellular virus-sensing system to regulate type I interferon (IFN) production, which is independent of toll-like receptors (TLR) (Yoneyama et al., 2005).

The antiviral role of RIG-I has been well recognized, and most studies focus on its activation mechanism and downstream signaling pathway in antiviral response. However, like many other proteins, RIG-I has multiple functions that are involved in a variety of cellular and physiological processes. This review discusses the recent studies on the role of RIG-I in antiviral response as well as in inflammation, apoptosis and development.

\section{SENSING VIRUS INFECTION}

Viruses are infectious pathogens and are extremely harmful for human health. They can utilize the replication machinery of the host to replicate themselves and amplify infection. As the first line of host defense, innate immunity has several sensors to detect viruses and produce type I IFN to limit the infection. RIG-I is one of such virus sensors that recognizes double stranded RNA (dsRNA) and induces IFN- $\beta$ production in a TLR-independent manner (Yoneyama et al., 2004). The different locations of TLRs and RLRs could detect different kinds of viruses. Unlike TLRs, which are membrane proteins and localized on the plasma membrane or endosome, RIG-I and other RLR family members are localized in cytoplasm and sense intracellular viral RNA (Yoneyama et al., 2005).

\section{The structure and function of RIG-I}

RIG-I contains two CARDs at $\mathrm{N}$-terminus that holds the signaling activation property. Over-expression of these 
tandem domains can potently drive IFN- $\beta$ production in the absence of virus (Yoneyama et al., 2004; Saito et al., 2007). Recent RIG-I signaling reconstitution study shows that RIG-I CARD can also function as a receptor to bind the unanchored K63-linked unbiquitin chains in an RNA- and ATP-dependent manner, which is essential for the full activation of RIG-I (Zeng et al., 2010). In the middle of $\mathrm{N}$-terminal CARD domain and Cterminal repressor domain, there is a helicase domain that contains an ATP binding motif and a TAS motif. The ATP binding motif is essential for RIG-I signaling because K270A mutation at this region disrupts IFN- $\beta$ induction (Sumpter et al., 2005). The C-terminus of RIG-I contains a repression domain (Saito et al., 2007), also called regulatory domain (RD) (Cui et al., 2008). Over-expression of RD can inhibit the virus-induced IFN- $\beta$ production. Moreover, the crystal structure of RD indicates that RD binds RNA and the zinc binding site in this domain is essential for RIG-I signaling (Cui et al., 2008). These results are consistent with the structural study of RIG-I C-terminal domain (CTD) by nuclear magnetic resonance (NMR) (Takahasi et al., 2008).

RIG-I is initially reported to bind dsRNA, which is considered to be the mechanism to distinguish self RNA and non-self infection (Yoneyama et al., 2004). Further study indicates that the dsRNA mimic poly $(\mathrm{I}: \mathrm{C})$ can only activate MDA5 but not RIG-I (Gitlin et al., 2006; Kato et al., 2006), while long dsRNA is the ligand for RIG-I (Kato et al., 2006). However, influenza A virus only generates single stranded RNA (ssRNA) rather than dsRNA, and it can also activate RIG-I, indicating that there should be a more accurate mechanism for RIG-I to distinguish self and non-self RNA (Pichlmair et al., 2006). Recent study also showed that RIG-I can recognize 5'-triphosphate RNA. However, if the 5'triphosphate RNA is capped or modified by nucleoside, both types of post-transcriptional modifications fail to activate RIGI (Hornung et al., 2006).

RIG-I functions as a virus sensor in a certain type of cells, such as fibroblasts, macrophages, conventional dendritic cells (cDC), but not in plasmacytoid dentritic cells (pDC) which use TLR system instead of the RIG-I-like receptor (RLR) system for the antiviral activity (Kato et al., 2005). As RNA binding proteins, RLRs mainly sense RNA virus. However, RIG-I and MDA5 have distinct spectra of viral detection. RIG-I mainly senses paramyxovirus, vesicular stomatitis virus (VSV) and influenza virus, while MDA5 mainly detects picornavirus (Kato et al., 2005; Kato et al., 2006).

\section{RIG-I signaling pathway}

Most knowledge of RIG-I signaling pathway comes from the related antiviral study. A CARD containing protein MAVS (also called VISA, IPS-1 or Cardif ) that acts as RIG-I downstream adaptor was independently identified by four groups (Kawai et al., 2005; Meylan et al., 2005; Seth et al., 2005; Xu et al., 2005). MAVS has an $\mathrm{N}$-terminal CARD domain, a proline-rich region (PRO) and a C-terminal trans-membrane domain (TM) that is required for its mitochondrial localization and signaling. The CARD domain is essential for MAVS to initiate cellular signaling and to interact with RIG-I. Moreover, TM is also required for its signaling, and TM deletion results in the detaining of MAVS in cytosol and loss of its activity (Seth et al., 2005).

RIG-I pathway is further divided into two branches that activate IRF3 and NF-KB, respectively. MITA (also called STING) was identified as a downstream adaptor of MAVS (Ishikawa and Barber, 2008; Zhong et al., 2008). After binding MITA, MAVS recruits TBK1 and IKKE to phosphorylate IRF3 and IRF7. Activated IRF3 and IRF7 form either homo-dimers or hetero-dimers, and enter the nucleus to initiate type I IFN transcription. On the other hand, MAVS also binds TRAF6, FADD and RIP1, recruits IKKa, IKK 3 and IKKY, and finally activates NF-KB (Seth et al., 2005). Shorter form of zinc-finger $\mathrm{CCCCH}$-type antiviral protein 1 (ZAPS) is a newly identified protein that associates with RIG-I and promotes RIG-I activity. Disruption of ZAPS causes impaired induction of IFNs and other cytokines, indicating that it is a key stimulatory factor of RIG-I (Hayakawa et al., 2011).

\section{The regulation of RIG-I signaling}

To avoid exaggerated IFN production, RIG-I signaling pathway is strictly regulated by several cellular mechanisms. LGP2 is the first molecule identified to suppress RIG-I signaling by competitively sequestrating dsRNA (Komuro and Horvath, 2006). However, genetic study demonstrates that LPG2 facilitates or enhances RIG-I signaling in certain virus infection (Venkataraman et al., 2007; Satoh et al., 2010). NS1 protein of influenza A virus inhibits RIG-I signaling through the interaction with RIG-I (Mibayashi et al., 2007). Autophage related protein Atg5-Atg12 complex and NLRC5 directly bind RIG-I and block the signaling (Jounai et al., 2007; Cui et al., 2010). NLRX1, gC1qR and PSMA7 are three newly identified proteins that inhibit RIG-I signaling (Moore et al., 2008; Jia et al., 2009; Xu et al., 2009). NLRX1 and gC1qR are localized on the outer membrane of mitochondria and interact with MAVS upon viral infection (Moore et al., 2008; Xu et al., 2009). NS3/4A of hepatitis $C$ virus (HCV) cleaves MAVS at C508 and drives the export of MAVS from mitochondria, leading to inactivation of MAVS protein (Lin et al., 2006). A20, SIKE and FLN29 suppress RIG-I signaling through the interaction with other downstream molecules (Huang et al., 2005; Saitoh et al., 2005; Sanada et al., 2008).

Several post-translational modifications of RIG-I, including ubiquitylation, phosphorylation and SUMOylation, also regulate RIG-I signaling. The E3 ligase TRIM25 interacts with RIG-I and efficiently delivers the Lys63-linked ubiquitin moiety to its CARD, leading to increased activity of RIG-I signaling (Gack et al., 2007). Riplet/RNF135 ubiquitinates RIG-I and promotes IFN production both in vitro and in vivo (Oshiumi 
et al., 2009, 2010). RNF125 is another ubiquitin E3 ligase that binds CARD and helicase domain of RIG-I and enhances the Lys48-linked ubiquitinization to cause RIG-I degradation (Arimoto et al., 2007). CYLD and DUBA are two RIG-I inhibitors with deubiquitinization activity, and both remove the Lys63-linked ubiquitin from RIG-I and TRAF3, respectively (Kayagaki et al., 2007; Friedman et al., 2008; Zhang et al., 2008a). A linear ubiquitin assembly complex (LUBAC) of HOIL-1L and HOIP specifically suppresses RIG-I activation by inducing TRIM25 degradation (Inn et al., 2011). Unlike most phosphorylation regulations, phosphorylated RIG-I is barely activated. Casein kinase 2 (CK2) phosphorylates RIG-I at T770, S854 and S855 in resting cells, which results in RIG-I inactivation. Once infected by RNA virus, but not DNA virus, these sites are dephosphorylated and RIG-I is consequently activated (Sun et al., 2011). Further study indicates that S8 and T170 of RIG-I are two other phosphorylation sites to keep RIG-I latent (Gack et al., 2010; NistalVillán et al., 2010). In addition to ubiquitylation and phosphorylation, recent study reports that RIG-I is also modified by small ubiquitin-like modifier-1 (SUMO-1), which enhances type I IFN production (Mi et al., 2010).

\section{RIG-I AND INFLAMMATION}

Inflammation is a defensive reaction for the host to remove harmful infection or repair damaged tissues. Acute inflammation is usually triggered by micro-organism infection and tissue injury. On the contrary, chronic inflammation is often accompanied with a variety of chronic diseases, such as type 2 diabetes and cardiovascular diseases (Medzhitov, 2008). In addition to its role in anti-viral immunity, emerged evidences also show that RIG-I participates in both acute and chronic inflammation.

\section{Possible roles of RIG-I in inflammation and inflammation related diseases}

Lipopolysaccharides (LPS) are large molecules localized at the outer membrane of Gram-negative bacteria, and they act as endotoxin to induce acute inflammation response by binding to TLR4 complex (Poltorak et al., 1998). Interestingly, RIG-I is found to be induced in LPS-stimulated endothelial cells. Moreover, over-expression of RIG-I also up-regulates cyclooxygenase-2 (COX-2) expression, indicating that RIG-I may have an important role in LPS-induced acute inflammation (Imaizumi et al., 2002). Several studies reveal that IFN-Y induces the expression of RIG-I in a variety of cell types, including human umbilical vein endothelial cells (HUVEC), vascular smooth muscle cells (SMC), urinary bladder epithelial cells, bronchial epithelial cells and pericardial mesothelial cells (Imaizumi et al., 2004a, 2004b, 2004c, 2005; Hatakeyama et al., 2007). In addition to IFN-y, several pro-inflammatory factors are able to stimulate RIG-I expression. TNF- $\alpha$ and IFN- $\gamma$ up-regulate RIG-I expression in keratinocytes, which is believed to be involved in psoriasis vulgaris (Kitamura et al., 2007). TNF- $\alpha$ alone can increase RIG-I expression in fibroblast-like synoviocytes in an IFN- $\beta$ dependent manner (Imaizumi et al., 2009). Interleukin (IL)-1 $\beta$ drives the expression of RIG-I in human gingival fibroblasts (Sakaki et al., 2005). All these studies indicate that RIG-I is involved in the regulation of inflammation.

Higher level of RIG-I has been found in several inflammation related diseases, indicating its possible modulatory role in inflammation. Atherosclerosis is considered as an inflammatory disease, and the intimal macrophages in atherosclerotic lesions contain high level of RIG-I protein, suggesting that RIG-I may be involved in the activation of macrophage during atherosclerotic genesis (Imaizumi et al., 2007). In lupus nephritis patients, the expression level of RIG-I increases in the urinary sediment (Tsugawa et al., 2008; Imaizumi et al., 2010). Elevated RIG-I expression is also observed in synovial tissues of rheumatoid arthritis, suggesting a possible role of RIG-I in the pathogenesis of synovial inflammation (Imaizumi et al., 2008). Moreover, RIG-I knockout mice develop a colitislike phenotype and RIG-I protein is shown to regulate $\mathrm{T}$ cell activation (Wang et al., 2007).

\section{Regulatory mechanism of RIG-I in inflammation}

Compared to the antiviral research, the mechanism of inflammation modulatory role of RIG-I is less studied. Until recently, several groups uncover the regulatory mechanism of RIG-I in acute and chronic inflammation. As a result of NF-KB activation, RIG-I induces the expression of inflammatory factors and chemokines, including IL-1 $\beta$, IL-6, IL-8, IL-28, IL29 and RANTES, in response to inflammatory stimulations (Kubota et al., 2006; Matikainen et al., 2006; Yoshida et al., 2007). In macrophage, RIG-I is induced by LPS through TRIF pathway, which partially results from IFN- $\beta$ autocrine secretion. The induced RIG-I leads to activation of tumor necrosis factor (TNF)- $\alpha$ promoter and regulates LPS induced expression of TNF- $\alpha$ at late phase; thus, it acts as a key factor in the auto-loop cascade for the amplification of inflammatory factors (Wang et al., 2008). Our latest study reveals a novel role of RIG-I in senescence associated inflammation (Liu et al., 2011). Senescent cells secrete a spectrum of proinflammatory factors and chemokines, such as IL-6 and IL8 , which is called senescence associated secretory phenotype (SASP) (Coppé et al., 2008). The expression of RIG-I increases with cell passages through a mechanism relying on ATM-IRF1 activation. RIG-I mediated senescence associated inflammation requires its downstream adaptor MAVS, which activates NF-KB and AP-1 to induce the expression of inflammatory factors. An anti-ageing protein klotho suppresses senescence associated inflammation by directly interacting with RIG-I and blocking its multimer formation during senescence (Liu et al., 2011). Block of RIG-I signaling 
not only inhibits senescence associated inflammation but also prolongs cell growth. It should be noted that RIG-I induced by non-viral factors only promotes the expression of inflammatory factors, but not the production of IFN- $\beta$. Therefore, the activation of NF-KB and IFN-stimulated response element (ISRE) by RIG-I should be regulated by different mechanisms.

\section{OTHER POSSIBLE ROLES OF RIG-I}

RIG-I was initially found as an inducible gene in a leukemia cell line treated with ATRA, which is an acknowledged agent to promote cell differentiation and widely used as an anticancer drug for leukemia (Liu et al., 2000). Therefore, it is possible that RIG-I is involved in some other cellular events such as carcinogenesis, apoptosis, senescence, cell differentiation and development.

\section{RIG-I and cancer}

It has been demonstrated that the level of RIG-I is quite low, even undetectable in most cancer cells such as prostate, breast, melanocyte and astrocyte malignant cells. However, RIG-I can be easily detected in their counterparts of normal cells (Su et al., 2007). RIG-I can be induced by IFN- $\gamma$, an immuno-modulatory factor, in human breast cancer cell MCF7 and cervical cancer cell HeLa. Furthermore, RIG-I mediates the IFN-y stimulation of ISG15 and CXCL11 in these cells, suggesting that RIG-I is involved in the immuno-modulatory function (Cui et al., 2004; Yuzawa et al., 2008). A micro-array screen of MDA-MB-435 human breast cancer cells showed that RIG-I is up-regulated in retinoic acid receptor $\beta 2$ (RARß2) transfected cells, indicating a possible role of RIG-I in antimetastasis (Wallden et al., 2005). Viral infection can lead to carcinogenesis. As a viral RNA receptor, RIG-I and its signaling molecules are frequently targeted by different components of virus, which leads to virus-induced progression of malignant mesothelioma by increasing vascular endothelial growth factor (VEGF) production (Wörnle et al., 2009). These studies provide novel insight into the role of RIG-I in cancer development.

\section{RIG-I and apoptosis}

It has been reported that RIG-I is involved in the apoptosis induced by poly $(\mathrm{I}: \mathrm{C})$ or synthetic retinoid in hepatoma or melanoma cells (Pan et al., 2009; Peng et al., 2009). However, the underlying mechanism is poorly understood. IFN- $\beta$ has a great potential to induce apoptosis. Although RIG-I signaling triggers the production of IFN- $\beta$, some reports show that RIG-I and MDA5 initiate a pro-apoptotic pathway independent of type I IFN. Noxa and downstream caspase-9, as well as Apaf-1, but not p53, are required for RIG-I induced apoptosis in melanoma cells, while in non-malignant cells, the
RIG-I induced apoptosis can be blocked by the anti-apoptotic protein Bcl-xL (Besch et al., 2009).

\section{RIG-I in development and proliferation}

The involvement of RIG-I in development and proliferation mainly relies on the finding that RIG-I can be induced by ATRA in leukemia. However, in normal myelopoiesis without retinoic acid stimulation, RIG-I expression also increased in culture, suggesting a possible role of RIG-I in granulocytic differentiation. RIG-I deficient mice exhibit developmental disorder in myeloproliferation due to the down-regulation of IFN consensus sequence binding protein (Zhang et al., 2008b). Another recent study reveals a novel RIG-I pathway in regulating cell proliferation. Under the regulation of STAT1, RIG-I conversely augments the activation of STAT1 on ISG expression to inhibit the proliferation of leukemia cells, which is independent of MAVS signaling (Jiang et al., 2011).

\section{RIG-I in phagocytosis}

It is shown that RIG-I has a pro-phagocytosis role in bacterial infection. Knockdown of RIG-I leads to defects in phagocytosis related pathway and inhibits LPS-induced phagocytosis in macrophage. The function of RIG-I in phagocytosis is independent of MAVS, indicating the different functions of RIG-I and its signaling. RIG-I deficient mice are more susceptible to Escherichia coli infection, suggesting that RIG-I might also participate in the anti-bacterial immunity (Kong et al., 2009).

\section{FUTURE PERSPECTIVES}

It is suggested that RIG-I is "born" to be a stress protein induced by retinoic acid, just as described by its name. The stimulations that up-regulate RIG-I expression include double/single stranded RNA in virus infection (Kubota et al., 2006), LPS in bacterial infection (Imaizumi et al., 2002), interferons (Imaizumi et al., 2004a), TNF- $\alpha$ (Imaizumi et al., 2009), oxidized cholesterol (unpublished data) and even DNA damage in cell senescence (Liu et al., 2011). In addition, the expression of RIG-I is shown to be up-regulated in a series of chronic inflammatory diseases, such as atherosclerosis (Imaizumi et al., 2007) and arthritis (Imaizumi et al., 2008). To more accurately define the characteristics of RIG-I, we would rather call it a "stress" protein -it is inducible and activated under different "stresses." Nevertheless, compared to the role of RIG-I in antiviral response, the knowledge about RIG-I in other fields is limited. Extensive studies are required to answer the questions about the role of RIG-I in non-viral conditions. For example, how is RIG-I activated in non-RNA systems, such as inflammation and cell proliferation? Future studies may focus on the mechanisms of RNA-independent RIG-I activation. 


\section{ABBREVIATIONS}

AP-1, activator protein 1; Apaf-1, apoptotic protease activating factor 1; ATM, ataxia telangiectasia mutated; ATP, adenosine triphosphate; $\mathrm{Bcl}-\mathrm{xL}$, B-cell lymphoma-extra large; Cardif, CARD adaptor inducing IFN- $\beta$; Caspase, cysteine-aspartic proteases; $C X C L$, chemokine (C$\mathrm{X}-\mathrm{C}$ motif) ligand; CYLD, cylindromatosis; DUBA, deubiquitinating enzyme $A ; F A D D$, Fas associated protein with death domain; $g C 1 q R$, receptor for global domain of complement $1 \mathrm{q}$ subunit; IKK, IKB kinase; IPS-1, IFN- $\beta$ promoter stimulator 1 ; IRF, interferon regulatory factor; ISG, interferon stimulated gene; MITA, mediator of IRF3 activation; NF-kB, nuclear factor KB; NLRC5, NOD-like receptor family CARD domain containing 5 ; NLRX1, NOD-like receptor family member X1; poly (I:C), polyriboinosinic:polyribocytidylic; PSMA7, proteasome subunit alpha type-7; RIP, receptor interacting protein; RNF, RING finger protein; RANTES, regulated upon activation, normal T-cell expressed, and secreted; SIKE, suppressor of IKKE; STAT, signal transducer and activator of transcription; STING, stimulator of interferon genes; TBK1, TANK binding kinase 1; TRAF, tumor necrosis factor receptor associated factor; TRIF, TIR domain containing adaptor protein inducing IFN- $\beta$; TRIM25, tripartite motifcontaining protein 25 ; VISA, virus induced signaling adaptor

\section{REFERENCES}

Arimoto, K., Takahashi, H., Hishiki, T., Konishi, H., Fujita, T., and Shimotohno, K. (2007). Negative regulation of the RIG-I signaling by the ubiquitin ligase RNF125. Proc Natl Acad Sci U S A 104, 7500-7505.

Besch, R., Poeck, H., Hohenauer, T., Senft, D., Häcker, G., Berking, C., Hornung, V., Endres, S., Ruzicka, T., Rothenfusser, S., et al. (2009). Proapoptotic signaling induced by RIG-I and MDA-5 results in type I interferon-independent apoptosis in human melanoma cells. J Clin Invest 119, 2399-2411.

Coppé, J.P., Patil, C.K., Rodier, F., Sun, Y., Muñoz, D.P., Goldstein, J., Nelson, P.S., Desprez, P.Y., and Campisi, J. (2008). Senescenceassociated secretory phenotypes reveal cell-nonautonomous functions of oncogenic RAS and the p53 tumor suppressor. PLoS Biol 6, 2853-2868.

Cui, J., Zhu, L., Xia, X., Wang, H.Y., Legras, X., Hong, J., Ji, J., Shen, P., Zheng, S., Chen, Z.J., et al. (2010). NLRC5 negatively regulates the NF-kappaB and type I interferon signaling pathways. Cell 141, 483- 496.

Cui, S., Eisenächer, K., Kirchhofer, A., Brzózka, K., Lammens, A., Lammens, K., Fujita, T., Conzelmann, K.K., Krug, A., and Hopfner, K.P. (2008). The C-terminal regulatory domain is the RNA 5'triphosphate sensor of RIG-I. Mol Cell 29, 169-179.

Cui, X.F., Imaizumi, T., Yoshida, H., Borden, E.C., and Satoh, K. (2004). Retinoic acid-inducible gene-I is induced by interferongamma and regulates the expression of interferon-gamma stimulated gene 15 in MCF-7 cells. Biochem Cell Biol 82, 401-405.

Friedman, C.S., O'Donnell, M.A., Legarda-Addison, D., Ng, A., Cárdenas, W.B., Yount, J.S., Moran, T.M., Basler, C.F., Komuro, A., Horvath, C.M., et al. (2008). The tumour suppressor CYLD is a negative regulator of RIG-I-mediated antiviral response. EMBO Rep 9, 930-936.

Gack, M.U., Nistal-Villán, E., Inn, K.S., García-Sastre, A., and Jung, J. U. (2010). Phosphorylation-mediated negative regulation of RIG-I antiviral activity. J Virol 84, 3220-3229.
Gack, M.U., Shin, Y.C., Joo, C.H., Urano, T., Liang, C., Sun, L., Takeuchi, O., Akira, S., Chen, Z., Inoue, S., et al. (2007). TRIM25 RING-finger E3 ubiquitin ligase is essential for RIG-I-mediated antiviral activity. Nature 446, 916-920.

Gitlin, L., Barchet, W., Gilfillan, S., Cella, M., Beutler, B., Flavell, R.A., Diamond, M.S., and Colonna, M. (2006). Essential role of mda-5 in type I IFN responses to polyriboinosinic:polyribocytidylic acid and encephalomyocarditis picornavirus. Proc Natl Acad Sci U S A 103, 8459-8464.

Hatakeyama, M., Imaizumi, T., Terasaki, F., Mori, F., Tanji, K., Sato, F., Kijima, H., Suma, H., Wakabayashi, K., Yoshida, H., et al. (2007). Interferon-gamma upregulates retinoic acid-inducible gene-I in human pericardial mesothelial cells. Acta Cardiol 62, 553-557.

Hayakawa, S., Shiratori, S., Yamato, H., Kameyama, T., Kitatsuji, C., Kashigi, F., Goto, S., Kameoka, S., Fujikura, D., Yamada, T., et al. (2011). ZAPS is a potent stimulator of signaling mediated by the RNA helicase RIG-I during antiviral responses. Nat Immunol 12, 37-44.

Hornung, V., Ellegast, J., Kim, S., Brzózka, K., Jung, A., Kato, H., Poeck, H., Akira, S., Conzelmann, K.K., Schlee, M., et al. (2006). 5'-Triphosphate RNA is the ligand for RIG-I. Science 314, 994-997.

Huang, J., Liu, T., Xu, L.G., Chen, D., Zhai, Z., and Shu, H.B. (2005). SIKE is an IKK epsilon/TBK1-associated suppressor of TLR3- and virus-triggered IRF-3 activation pathways. EMBO J 24, 4018-4028.

Imaizumi, T., Aratani, S., Nakajima, T., Carlson, M., Matsumiya, T., Tanji, K., Ookawa, K., Yoshida, H., Tsuchida, S., McIntyre, T.M., et al. (2002). Retinoic acid-inducible gene-I is induced in endothelial cells by LPS and regulates expression of COX-2. Biochem Biophys Res Commun 292, 274-279.

Imaizumi, T., Arikawa, T., Sato, T., Uesato, R., Matsumiya, T., Yoshida, H., Ueno, M., Yamasaki, S., Nakajima, T., Hirashima, M., et al. (2008). Involvement of retinoic acid-inducible gene-I in inflammation of rheumatoid fibroblast-like synoviocytes. Clin Exp Immunol 153, 240-244.

Imaizumi, T., Hatakeyama, M., Yamashita, K., Yoshida, H., Ishikawa, A., Taima, K., Satoh, K., Mori, F., and Wakabayashi, K. (2004a). Interferon-gamma induces retinoic acid-inducible gene-I in endothelial cells. Endothelium 11, 169-173.

Imaizumi, T., Kumagai, M., Taima, K., Fujita, T., Yoshida, H., and Satoh, K. (2005). Involvement of retinoic acid-inducible gene-I in the IFN-gamma/STAT1 signalling pathway in BEAS-2B cells. Eur Respir J 25, 1077-1083.

Imaizumi, T., Matsumiya, T., Yoshida, H., Naraoka, T., Uesato, R., Ishibashi, Y., Ota, K., Toh, S., Fukuda, S., and Satoh, K. (2009). Tumor-necrosis factor-alpha induces retinoic acid-inducible gene-I in rheumatoid fibroblast-like synoviocytes. Immunol Lett 122, 89-93.

Imaizumi, T., Tanaka, H., Tajima, A., Tsuruga, K., Oki, E., Sashinami, H., Matsumiya, T., Yoshida, H., Inoue, I., and Ito, E. (2010). Retinoic acid-inducible gene-I (RIG-I) is induced by IFN-gamma in human mesangial cells in culture: possible involvement of RIG-I in the inflammation in lupus nephritis. Lupus 19, 830-836.

Imaizumi, T., Yagihashi, N., Hatakeyama, M., Yamashita, K., Ishikawa, A., Taima, K., Yoshida, H., Inoue, I., Fujita, T., Yagihashi, S., et al. (2004b). Expression of retinoic acid-inducible gene-l in vascular smooth muscle cells stimulated with interferon-gamma. Life Sci 75, 1171-1180.

Imaizumi, T., Yagihashi, N., Hatakeyama, M., Yamashita, K., 
Ishikawa, A., Taima, K., Yoshida, H., Yagihashi, S., and Satoh, K. (2004c). Upregulation of retinoic acid-inducible gene-I in T24 urinary bladder carcinoma cells stimulated with interferon-gamma. Tohoku J Exp Med 203, 313-318.

Imaizumi, T., Yagihashi, N., Kubota, K., Yoshida, H., Sakaki, H., Yagihashi, S., Kimura, H., and Satoh, K. (2007). Expression of retinoic acid-inducible gene-I (RIG-I) in macrophages: possible involvement of RIG-I in atherosclerosis. J Atheroscler Thromb 14, $51-55$.

Inn, K.S., Gack, M.U., Tokunaga, F., Shi, M., Wong, L.Y., Iwai, K., and Jung, J.U. (2011). Linear ubiquitin assembly complex negatively regulates RIG-I- and TRIM25-mediated type I interferon induction. Mol Cell 41, 354-365.

Ishikawa, H., and Barber, G.N. (2008). STING is an endoplasmic reticulum adaptor that facilitates innate immune signalling. Nature 455, 674-678.

Jia, Y., Song, T., Wei, C., Ni, C., Zheng, Z., Xu, Q., Ma, H., Li, L., Zhang, Y., He, X., et al. (2009). Negative regulation of MAVSmediated innate immune response by PSMA7. J Immunol 183, 4241-4248.

Jiang, L.J., Zhang, N.N., Ding, F., Li, X.Y., Chen, L., Zhang, H.X., Zhang, W., Chen, S.J., Wang, Z.G., Li, J.M., et al. (2011). RAinducible gene-I induction augments STAT1 activation to inhibit leukemia cell proliferation. Proc Natl Acad Sci U S A 108, 1897-1902.

Jounai, N., Takeshita, F., Kobiyama, K., Sawano, A., Miyawaki, A., Xin, K.Q., Ishii, K.J., Kawai, T., Akira, S., Suzuki, K., et al. (2007). The Atg5 Atg12 conjugate associates with innate antiviral immune responses. Proc Natl Acad Sci U S A 104, 14050-14055.

Kato, H., Sato, S., Yoneyama, M., Yamamoto, M., Uematsu, S., Matsui, K., Tsujimura, T., Takeda, K., Fujita, T., Takeuchi, O., et al. (2005). Cell type-specific involvement of RIG-I in antiviral response. Immunity 23, 19-28.

Kato, H., Takeuchi, O., Sato, S., Yoneyama, M., Yamamoto, M., Matsui, K., Uematsu, S., Jung, A., Kawai, T., Ishii, K.J., et al. (2006). Differential roles of MDA5 and RIG-I helicases in the recognition of RNA viruses. Nature 441, 101-105.

Kawai, T., Takahashi, K., Sato, S., Coban, C., Kumar, H., Kato, H., Ishii, K.J., Takeuchi, O., and Akira, S. (2005). IPS-1, an adaptor triggering RIG-I- and Mda5-mediated type I interferon induction. Nat Immunol 6, 981-988.

Kayagaki, N., Phung, Q., Chan, S., Chaudhari, R., Quan, C., O'Rourke, K.M., Eby, M., Pietras, E., Cheng, G., Bazan, J.F., et al. (2007). DUBA: a deubiquitinase that regulates type I interferon production. Science 318, 1628-1632.

Kitamura, H., Matsuzaki, Y., Kimura, K., Nakano, H., Imaizumi, T., Satoh, K., and Hanada, K. (2007). Cytokine modulation of retinoic acid-inducible gene-I (RIG-I) expression in human epidermal keratinocytes. J Dermatol Sci 45, 127-134.

Komuro, A., and Horvath, C.M. (2006). RNA- and virus-independent inhibition of antiviral signaling by RNA helicase LGP2. J Virol 80, 12332-12342.

Kong, L., Sun, L., Zhang, H., Liu, Q., Liu, Y., Qin, L., Shi, G., Hu, J.H., $\mathrm{Xu}$, A., Sun, Y.P., et al. (2009). An essential role for RIG-I in toll-like receptor-stimulated phagocytosis. Cell Host Microbe 6, 150-161.

Kubota, K., Sakaki, H., Imaizumi, T., Nakagawa, H., Kusumi, A., Kobayashi, W., Satoh, K., and Kimura, H. (2006). Retinoic acidinducible gene-I is induced in gingival fibroblasts by lipopolysaccharide or poly IC: possible roles in interleukin-1 beta, -6 and -8 expression. Oral Microbiol Immunol 21, 399-406.

Lin, R., Lacoste, J., Nakhaei, P., Sun, Q., Yang, L., Paz, S., Wilkinson, P., Julkunen, I., Vitour, D., Meurs, E., et al. (2006). Dissociation of a MAVS/IPS-1/VISA/Cardif-IKKepsilon molecular complex from the mitochondrial outer membrane by hepatitis $C$ virus NS3-4A proteolytic cleavage. J Virol 80, 6072-6083.

Liu, F., Wu, S., Ren, H., and Gu, J. (2011). Klotho suppresses RIG-Imediated senescence-associated inflammation. Nat Cell Biol 13, 254-262.

Liu, T.X., Zhang, J.W., Tao, J., Zhang, R.B., Zhang, Q.H., Zhao, C.J., Tong, J.H., Lanotte, M., Waxman, S., Chen, S.J., et al. (2000). Gene expression networks underlying retinoic acid-induced differentiation of acute promyelocytic leukemia cells. Blood 96, 1496-1504.

Matikainen, S., Sirén, J., Tissari, J., Veckman, V., Pirhonen, J., Severa, M., Sun, Q., Lin, R., Meri, S., Uzé, G., et al. (2006). Tumor necrosis factor alpha enhances influenza A virus-induced expression of antiviral cytokines by activating RIG-I gene expression. J Virol 80, 3515-3522.

Medzhitov, R. (2008). Origin and physiological roles of inflammation. Nature 454, 428-435.

Meylan, E., Curran, J., Hofmann, K., Moradpour, D., Binder, M., Bartenschlager, R., and Tschopp, J. (2005). Cardif is an adaptor protein in the RIG-I antiviral pathway and is targeted by hepatitis $\mathrm{C}$ virus. Nature 437, 1167-1172.

Mi, Z., Fu, J., Xiong, Y., and Tang, H. (2010). SUMOylation of RIG-I positively regulates the type I interferon signaling. Protein Cell 1 , 275-283.

Mibayashi, M., Martínez-Sobrido, L., Loo, Y.M., Cárdenas, W.B., Gale, M. Jr, and García-Sastre, A. (2007). Inhibition of retinoic acidinducible gene I-mediated induction of beta interferon by the NS1 protein of influenza A virus. J Virol 81, 514-524.

Moore, C.B., Bergstralh, D.T., Duncan, J.A., Lei, Y., Morrison, T.E., Zimmermann, A.G., Accavitti-Loper, M.A., Madden, V.J., Sun, L., Ye, Z., et al. (2008). NLRX1 is a regulator of mitochondrial antiviral immunity. Nature 451, 573-577.

Nistal-Villán, E., Gack, M.U., Martínez-Delgado, G., Maharaj, N.P., Inn, K.S., Yang, H., Wang, R., Aggarwal, A.K., Jung, J.U., and García-Sastre, A. (2010). Negative role of RIG-I serine 8 phosphorylation in the regulation of interferon-beta production. $\mathrm{J}$ Biol Chem 285, 20252-20261.

Oshiumi, H., Matsumoto, M., Hatakeyama, S., and Seya, T. (2009). Riplet/RNF135, a RING finger protein, ubiquitinates RIG-I to promote interferon-beta induction during the early phase of viral infection. J Biol Chem 284, 807-817.

Oshiumi, H., Miyashita, M., Inoue, N., Okabe, M., Matsumoto, M., and Seya, T. (2010). The ubiquitin ligase Riplet is essential for RIG-Idependent innate immune responses to RNA virus infection. Cell Host Microbe 8, 496-509.

Pan, M., Geng, S., Xiao, S., Ren, J., Liu, Y., Li, X., Li, Z., and Peng, Z. (2009). Apoptosis induced by synthetic retinoic acid CD437 on human melanoma A375 cells involves RIG-I pathway. Arch Dermatol Res 301, 15-20.

Peng, S., Geng, J., Sun, R., Tian, Z., and Wei, H. (2009). Polyinosinicpolycytidylic acid liposome induces human hepatoma cells apoptosis which correlates to the up-regulation of RIG-I like receptors. Cancer Sci 100, 529-536. 
Pichlmair, A., Schulz, O., Tan, C.P., Näslund, T.I., Liljeström, P., Weber, F., and Reis e Sousa, C. (2006). RIG-I-mediated antiviral responses to single-stranded RNA bearing 5'-phosphates. Science 314, 997-1001.

Poltorak, A., He, X., Smirnova, I., Liu, M.Y., Van Huffel, C., Du, X., Birdwell, D., Alejos, E., Silva, M., Galanos, C., et al. (1998). Defective LPS signaling in $\mathrm{C} 3 \mathrm{H} / \mathrm{HeJ}$ and $\mathrm{C} 57 \mathrm{BL} / 10 \mathrm{ScCr}$ mice: mutations in Tlr4 gene. Science 282, 2085-2088.

Saito, T., Hirai, R., Loo, Y.M., Owen, D., Johnson, C.L., Sinha, S.C., Akira, S., Fujita, T., and Gale, M. Jr. (2007). Regulation of innate antiviral defenses through a shared repressor domain in RIG-I and LGP2. Proc Natl Acad Sci U S A 104, 582-587.

Saitoh, T., Yamamoto, M., Miyagishi, M., Taira, K., Nakanishi, M., Fujita, T., Akira, S., Yamamoto, N., and Yamaoka, S. (2005). A20 is a negative regulator of IFN regulatory factor 3 signaling. J Immunol 174, 1507-1512.

Sakaki, H., Imaizumi, T., Matsumiya, T., Kusumi, A., Nakagawa, H., Kubota, K., Nishi, N., Nakamura, T., Hirashima, M., Satoh, K., et al. (2005). Retinoic acid-inducible gene-I is induced by interleukin1 beta in cultured human gingival fibroblasts. Oral Microbiol Immunol 20, 47-50.

Sanada, T., Takaesu, G., Mashima, R., Yoshida, R., Kobayashi, T., and Yoshimura, A. (2008). FLN29 deficiency reveals its negative regulatory role in the Toll-like receptor (TLR) and retinoic acidinducible gene I (RIG-I)-like helicase signaling pathway. J Biol Chem 283, 33858-33864.

Satoh, T., Kato, H., Kumagai, Y., Yoneyama, M., Sato, S., Matsushita, K., Tsujimura, T., Fujita, T., Akira, S., and Takeuchi, O. (2010). LGP2 is a positive regulator of RIG-I- and MDA5-mediated antiviral responses. Proc Natl Acad Sci U S A 107, 1512-1517.

Seth, R.B., Sun, L., Ea, C.K., and Chen, Z.J. (2005). Identification and characterization of MAVS, a mitochondrial antiviral signaling protein that activates NF-kappaB and IRF 3. Cell 122, 669-682.

Su, Z.Z., Sarkar, D., Emdad, L., Barral, P.M., and Fisher, P.B. (2007). Central role of interferon regulatory factor-1 (IRF-1) in controlling retinoic acid inducible gene-I (RIG-I) expression. J Cell Physiol 213, 502-510.

Sumpter, R. Jr, Loo, Y.M., Foy, E., Li, K., Yoneyama, M., Fujita, T., Lemon, S.M., and Gale, M. Jr. (2005). Regulating intracellular antiviral defense and permissiveness to hepatitis $C$ virus RNA replication through a cellular RNA helicase, RIG-I. J Virol 79, 2689-2699.

Sun, Z., Ren, H., Liu, Y., Teeling, J.L., and Gu, J. (2011). Phosphorylation of RIG-I by casein kinase II inhibits its antiviral response. J Virol 85, 1036-1047.

Takahasi, K., Yoneyama, M., Nishihori, T., Hirai, R., Kumeta, H., Narita, R., Gale, M. Jr, Inagaki, F., and Fujita, T. (2008). Nonself RNA-sensing mechanism of RIG-I helicase and activation of antiviral immune responses. Mol Cell 29, 428-440.

Tsugawa, K., Oki, E., Suzuki, K., Imaizumi, T., Ito, E., and Tanaka, H. (2008). Expression of mRNA for functional molecules in urinary sediment in glomerulonephritis. Pediatr Nephrol 23, 395- 401.

Venkataraman, T., Valdes, M., Elsby, R., Kakuta, S., Caceres, G., Saijo, S., Iwakura, Y., and Barber, G.N. (2007). Loss of DExD/H box RNA helicase LGP2 manifests disparate antiviral responses. J Immunol 178, 6444-6455.

Wallden, B., Emond, M., Swift, M.E., Disis, M.L., and Swisshelm, K.
(2005). Antimetastatic gene expression profiles mediated by retinoic acid receptor beta 2 in MDA-MB-435 breast cancer cells. BMC Cancer 5, 140.

Wang, J., Wu, S., Jin, X., Li, M., Chen, S., Teeling, J.L., Perry, V.H., and $\mathrm{Gu}, J$. (2008). Retinoic acid-inducible gene-I mediates late phase induction of TNF-alpha by lipopolysaccharide. J Immunol 180, 8011-8019.

Wang, Y., Zhang, H.X., Sun, Y.P., Liu, Z.X., Liu, X.S., Wang, L., Lu, S. Y., Kong, H., Liu, Q.L., Li, X.H., et al. (2007). Rig-I-/- mice develop colitis associated with downregulation of $\mathrm{G}$ alpha i2. Cell Res 17, 858-868.

Wörnle, M., Sauter, M., Kastenmüller, K., Ribeiro, A., Roeder, M., Mussack, T., Ladurner, R., and Sitter, T. (2009). Role of viral induced vascular endothelial growth factor (VEGF) production in pleural effusion and malignant mesothelioma. Cell Biol Int 33, 180-186.

Xu, L., Xiao, N., Liu, F., Ren, H., and Gu, J. (2009). Inhibition of RIG-I and MDA5-dependent antiviral response by $\mathrm{gC} 1 \mathrm{qR}$ at mitochondria. Proc Natl Acad Sci U S A 106, 1530-1535.

Xu, L.G., Wang, Y.Y., Han, K.J., Li, L.Y., Zhai, Z., and Shu, H.B. (2005). VISA is an adapter protein required for virus-triggered IFNbeta signaling. Mol Cell 19, 727-740.

Yoneyama, M., Kikuchi, M., Matsumoto, K., Imaizumi, T., Miyagishi, M., Taira, K., Foy, E., Loo, Y.M., Gale, M. Jr, Akira, S., et al. (2005). Shared and unique functions of the DExD/H-box helicases RIG-I, MDA5, and LGP2 in antiviral innate immunity. J Immunol 175, 2851-2858.

Yoneyama, M., Kikuchi, M., Natsukawa, T., Shinobu, N., Imaizumi, T., Miyagishi, M., Taira, K., Akira, S., and Fujita, T. (2004). The RNA helicase RIG-I has an essential function in double-stranded RNAinduced innate antiviral responses. Nat Immunol 5, 730-737.

Yoshida, H., Imaizumi, T., Lee, S.J., Tanji, K., Sakaki, H., Matsumiya, T., Ishikawa, A., Taima, K., Yuzawa, E., Mori, F., et al. (2007). Retinoic acid-inducible gene-I mediates RANTES/CCL5 expression in U373MG human astrocytoma cells stimulated with doublestranded RNA. Neurosci Res 58, 199-206.

Yuzawa, E., Imaizumi, T., Matsumiya, T., Yoshida, H., Fukuhara, R., Kimura, H., Fukui, A., Tanji, K., Mori, F., Wakabayashi, K., et al. (2008). Retinoic acid-inducible gene-I is induced by interferongamma and regulates CXCL11 expression in HeLa cells. Life Sci 82, 670-675.

Zeng, W., Sun, L., Jiang, X., Chen, X., Hou, F., Adhikari, A., Xu, M., and Chen, Z.J. (2010). Reconstitution of the RIG-I pathway reveals a signaling role of unanchored polyubiquitin chains in innate immunity. Cell 141, 315-330.

Zhang, M., Wu, X., Lee, A.J., Jin, W., Chang, M., Wright, A., Imaizumi, T., and Sun, S.C. (2008a). Regulation of IkappaB kinase-related kinases and antiviral responses by tumor suppressor CYLD. J Biol Chem 283, 18621-18626.

Zhang, N.N., Shen, S.H., Jiang, L.J., Zhang, W., Zhang, H.X., Sun, Y. P., Li, X.Y., Huang, Q.H., Ge, B.X., Chen, S.J., et al. (2008b). RIG-I plays a critical role in negatively regulating granulocytic proliferation. Proc Natl Acad Sci U S A 105, 10553-10558.

Zhong, B., Yang, Y., Li, S., Wang, Y.Y., Li, Y., Diao, F., Lei, C., He, X., Zhang, L., Tien, P., et al. (2008). The adaptor protein MITA links virus-sensing receptors to IRF3 transcription factor activation. Immunity 29, 538-550. 